Check for updates

Cite this: Energy Adv., 2022,

1,9

Received 22nd September 2021, Accepted 21st October 2021

DOI: $10.1039 /$ d1ya00003a

rsc.li/energy-advances

\title{
Molybdenum as a versatile dopant in SnTe: a promising material for thermoelectric application
}

\author{
U. Sandhya Shenoy (D) *a and D. Krishna Bhat (D) *b
}

\begin{abstract}
The key to enhancing the thermoelectric performance of SnTe is to engineer its electronic structure by doping. It is essential that the beneficial features are exhibited at the Fermi level so as to exploit the benefits without the use of a co-dopant. We report molybdenum as such a versatile dopant in SnTe. The first-principles calculations reveal that $M o$ is able to introduce resonance levels as well as increase the band gap in SnTe. It not only causes the convergence of light and heavy hole valence sub-bands but also does so in the conduction band. The unique feature is the Rashba splitting of the conduction bands, leading to multiband transport. The transport properties calculated using Boltzmann transport equations predict the dual nature of the resonant dopant with a promising $Z T$ of $\sim 1.84$ and $\sim 1.1$ as a p- and an n-type dopant, respectively, in SnTe at $800 \mathrm{~K}$.
\end{abstract}

\section{Introduction}

Tin telluride is considered as a promising thermoelectric material whose performance can be further improvised in converting waste heat into useful electricity. ${ }^{1-4}$ For enhancing the figure of merit $(Z T)$ of a material, it is essential to increase the power factor $\left(\sigma S^{2}\right)$ and decrease the thermal conductivity $(\kappa)$ simultaneously. ${ }^{5,6}$ Moreover, choosing the right dopant is essential as electrical conductivity $(\sigma)$ and the Seebeck co-efficient $(S)$ are interrelated in a contrary way via carrier concentration. In addition to having an optimum carrier concentration, a material should also have an optimum band gap to act as a good thermoelectric. This is due to the fact that an appropriate band gap will prevent the bipolar diffusion of charge carriers due to thermal excitation at higher temperatures. ${ }^{7}$ Further, a band gap value higher than $6 k_{\mathrm{B}} T$ is preferred, which is $\sim 0.41 \mathrm{eV}$ at $800 \mathrm{~K}^{8}{ }^{8} \mathrm{SnTe}$ has a band gap of $0.18 \mathrm{eV}$ at $300 \mathrm{~K}$. It is well known that at higher

\footnotetext{
${ }^{a}$ Department of Chemistry, College of Engineering and Technology,

Srinivas University, Mukka, Mangalore, 574146, India.

E-mail: sandhyashenoy347@gmail.com

${ }^{b}$ Department of Chemistry, National Institute of Technology Karnataka, Surathkal,

Mangalore, 575025, India. E-mail: denthajekb@gmail.com
}

temperatures the band gap of SnTe increases due to the decrease in the valence band dispersion or bandwidth due to the decrease in the orbital overlap interaction as a consequence of the bond length increase due to thermal expansion. Also, the emphanitic behavior of the $\mathrm{Sn}^{2+}$ in SnTe decreases the energy of $\mathrm{s}^{2}$ lone pair states near the valence band edge, contributing further to the increasing band gap at higher temperatures. ${ }^{9}$ With increase in temperature the band gap of SnTe increases at a rate of $\sim 4 \times 10^{-4} \mathrm{eV} \mathrm{K}^{-1}$, which translates to a value of around $0.38 \mathrm{eV}$ at $800 \mathrm{~K}$, still lower than the required value and hence shows poor performance at high temperature. At high temperatures, the bipolar effect due to the low band gap is detrimental to the $Z T$ as the total ' $S$ ' value decreases due to the offsetting of the ' $S$ ' values contributed by the majority carriers by the ' $S$ ' values of opposite signs contributed by the minority carriers. ${ }^{7,10}$ It also causes an increase in ' $k$ ' values due to the heat carried by the charge carrier during ambipolar diffusion across the band gap. ${ }^{7,10}$ Hence, dopants like $\mathrm{Ag}, \mathrm{Ca}, \mathrm{Cd}, \mathrm{Mg}$, and $\mathrm{V}$ which can open the band gap help in improving the high temperature $Z T$ values. ${ }^{11-18}$ In addition, these dopants are also known to cause convergence of valence sub-bands, resulting in increased density of states effective masses of carriers and in turn increased ' $S$ ' values. ${ }^{13,16,18,19}$ Dopants which can distort the density of states near the Fermi level, i.e., resonant dopants, are known to improve the performance at lower temperatures. Bi, In, $\mathrm{Zn}, \mathrm{V}$ and $\mathrm{Mn}$ are known to introduce resonance levels in SnTe. ${ }^{20-24} \mathrm{~A}$ record high room temperature $Z T$ of 0.3 (@300 K) for lead free SnTe was reported by co-doping SnTe with Bi and Zn resonant dopants. ${ }^{24}$ To decrease the thermal conductivity, dopants like $\mathrm{Cu}, \mathrm{Sb}$ and $\mathrm{Pb}$ are known to be very effective. ${ }^{2,25-29}$ Co-doping and multi-doping with elements are also implemented to synergistically extract different benefits and increase the $Z T$ for a broad range of temperatures. ${ }^{2,12,13,16,20,23-31}$ Although resonant dopants improve the ' $S$ ' value, they decrease the ' $\sigma$ ' value due to carrier scattering and decreased mobility and hence exhibit poor power factor values. ${ }^{32}$ Many of the currently implemented techniques are good at improving the high temperature power factor, leading to a record value of $\sim 47 \mu \mathrm{W} \mathrm{cm}{ }^{-1} \mathrm{~K}^{-2}$ in SnTe 
co-doped with $\mathrm{Ca}$ and resonant dopant In, but exhibit poor values at room temperature. ${ }^{30} \mathrm{~A} Z T$ of $\sim 1.85$ at $823 \mathrm{~K}$ was achieved in SnTe by multidoping with $\mathrm{Ca}$, In and $\mathrm{Cu}$ but the average $Z T$ still remained at $0.67 .{ }^{31} \mathrm{~A}$ record high room temperature $Z T$ of 0.35 (@300 K) and a high average $Z T$ of $\sim 0.9$ between $300 \mathrm{~K}$ and $840 \mathrm{~K}$ were reported previously in SnTe by co-doping with $\mathrm{Pb}$ and resonant dopant $\mathrm{Zn}$ with a $Z T$ maximum of $\sim 1.66$ at $840 \mathrm{~K} .{ }^{28}$ Hence, it is the need of the day to find resonant dopants which can exhibit beneficial features to keep the power factor high enough both at low and at high temperatures, aiding the development of lead free thermoelectric materials.

Herein we report the realization of both $\mathrm{p}$ and $\mathrm{n}$ type thermoelectric properties by doping Mo into SnTe. We show here that Mo can significantly modify the electronic structure by introducing resonance states, which is essential for improving the low temperature performance. It also enlarges the band gap of the material and causes valence and conduction sub-band convergence essential for high temperature performance. ${ }^{13}$ The interesting feature is the multi-band transport resulting from the Rashba splitting of the conduction bands, leading to enhanced performance of the material. The transport property calculations based on the electronic structure predicted the material to be a potential high-performance material worth experimental investigation.

\section{Computational details}

The electronic structures of pristine SnTe and Mo doped SnTe were simulated using the open-source software Quantum ESPRESSO package. ${ }^{33}$ A $\sqrt{2} \times \sqrt{ } 2 \times 2$ supercell of a rock salt structure containing 32 atoms was used for the first-principles calculations. Pseudopotentials with a generalized gradient approximation to exchange-correlation energy with the Perdew, Burke, and Ernzerhof (PBE) functional type were used to treat the valence and semi-core electronic states. ${ }^{34}$ To involve the spin-orbit coupling interaction in the determination of their electronic structures, fully relativistic pseudopotentials considering Sn $\left(4 d^{10} 5 s^{2} 5 p^{2}\right)$, Te $\left(4 d^{10} 5 s^{2} 5 p^{4}\right)$ and Mo $\left(4 s^{2} 4 p^{6} 4 d^{5} 5 s^{1}\right)$ as valence electrons were used. It needs to be noted that the conventional
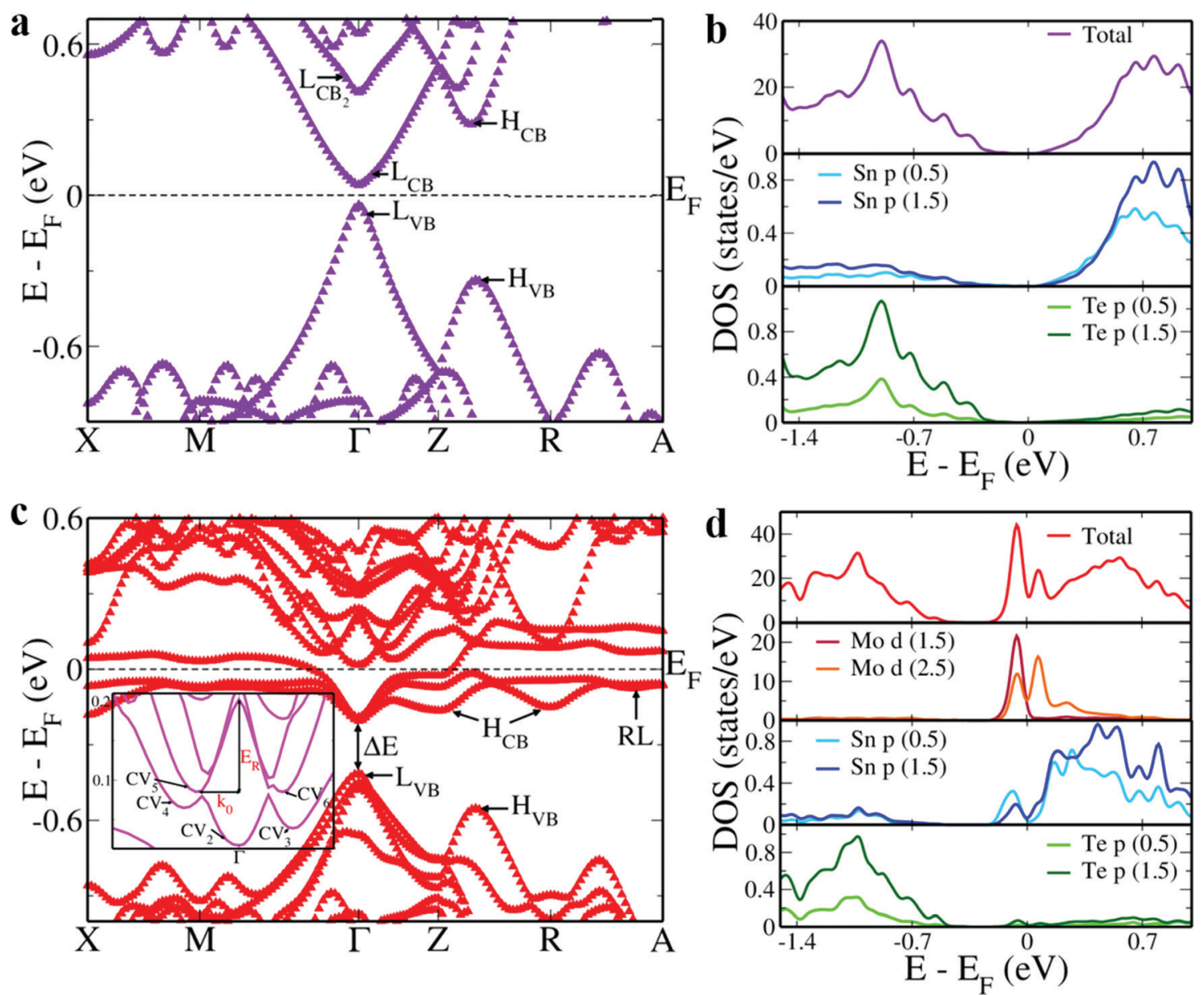

Fig. 1 Electronic structures and PDOS of $\mathrm{Sn}_{16} T_{16}\left(a\right.$ and $b$ ) and $\mathrm{Sn}_{15} \mathrm{MOTe}_{16}$ (c and d). $L_{\mathrm{VB}}, L_{\mathrm{CB}}, H_{\mathrm{VB}}$, and $H_{\mathrm{CB}}$ are the light hole valence band, light electron conduction band, heavy hole valence band and heavy electron conduction band respectively. $L_{\mathrm{CB}_{2}}$ refers to the second set of eight-fold degenerate light electron conduction bands at the $\Gamma$ point in $\mathrm{Sn}_{16} \mathrm{Te}_{16}$. RL refers to the resonance level. The inset of (c) shows the Rashba splitting occurring in the conduction band as a result of Mo doping. $\mathrm{CV}_{2}, \mathrm{CV}_{3}, \mathrm{CV}_{4}, \mathrm{CV}_{5}$, and $\mathrm{CV}_{6}$ indicate various valleys in the conduction band, potentially contributing to the transport properties. $E_{\mathrm{R}}$ refers to the Rashba energy and the momentum offset is indicated by $k_{0}$. 
GGA underestimates the band gap of the materials while incorporation of the Hubbard $U$ factor or hybrid functionals can give better band gap estimates. ${ }^{35,36}$ Nevertheless, the trend in the band gap predicted by the current method used is accurate. The total energy was determined using a $k$-mesh of $14 \times 14 \times 10$ points, and an energy and charge density cutoff of 50 Ry and 400 Ry, respectively.

\section{Results and discussion}

The electronic structure largely influences the power factor due to the competing traits of ' $\sigma$ ' and ' $S$ ' and hence plays a crucial role in improving the performance of thermoelectric materials. ${ }^{2,10,13} \mathrm{We}$ see an increase in the principal band gap $(\Delta E)$ of SnTe after Mo doping at the $\Gamma$ point from 0.08 to $0.21 \mathrm{eV}$ (Fig. 1). The appearance of a band gap at the $\Gamma$ point due to the folding of the $L$ point in the supercell and the underestimation of the band gap in DFT based calculations are well documented in previous reports. ${ }^{13,22,24,28,37}$ The large energy separation between the heavy hole $\left(H_{\mathrm{VB}}\right)$ and light hole $\left(L_{\mathrm{VB}}\right)$ valence bands of $0.30 \mathrm{eV}$ observed in SnTe is a matter of concern. ${ }^{24}$ As the Fermi level increases in energy with increase in temperature, it leads to the suppression of the contribution from the heavy hole valence band at the $Z+\delta$ point in the $Z \rightarrow R$ direction, resulting in decreased ' $S$ ' values. ${ }^{15}$
We observe that substitutional doping with Mo leads to a decrease in this energy offset to $0.14 \mathrm{eV}$, leading to an increase in the number of degenerate valleys, contributing to higher ' $S$ ' values. ${ }^{30}$ Such band convergence has a very little influence on carrier mobility and hence keeps the power factor high. ${ }^{19}$ Similarly, we estimate a decrease in energy offset in the conduction band from $0.24 \mathrm{eV}$ to $0.04 \mathrm{eV}$ and $0.05 \mathrm{eV}$ considering the heavy electron conduction sub-bands $\left(H_{\mathrm{CB}}\right)$ at the $Z$ point and $R$ point, respectively. This shift in the position of $H_{\mathrm{CB}}$ from the $Z+\delta$ point to the $Z$ and $R$ points is a consequence of alterations taking place in the higher energy conduction bands which is explained later. Another heavy carrier band is also generated at the $R$ point $0.1 \mathrm{eV}$ above the Fermi level. The density of states (DOS) reveals that in both doped and undoped SnTe the conduction bands are formed from $\mathrm{Sn}$ ' $\mathrm{p}$ ' orbitals and the valence bands from Te 'p' orbitals. The doped configuration in addition shows a well defined peak near the Fermi level formed due to the hybridization of Mo ' $d$ ' orbitals with Sn 'p' orbitals, indicating the formation of resonance levels. While in the case of $\mathrm{V}$ doped SnTe we see the contribution of the d orbitals with both $j$ values 1.5 and 2.5 at one point, here we see the d orbitals of Mo with a $j$ value of 2.5 giving rise to two peaks like a doublet. ${ }^{18}$ The eightfold degenerate conduction band loses its degeneracy with substitution of Mo in the Sn site and splits into four sets of doubly degenerate bands. The lowermost three sets form the
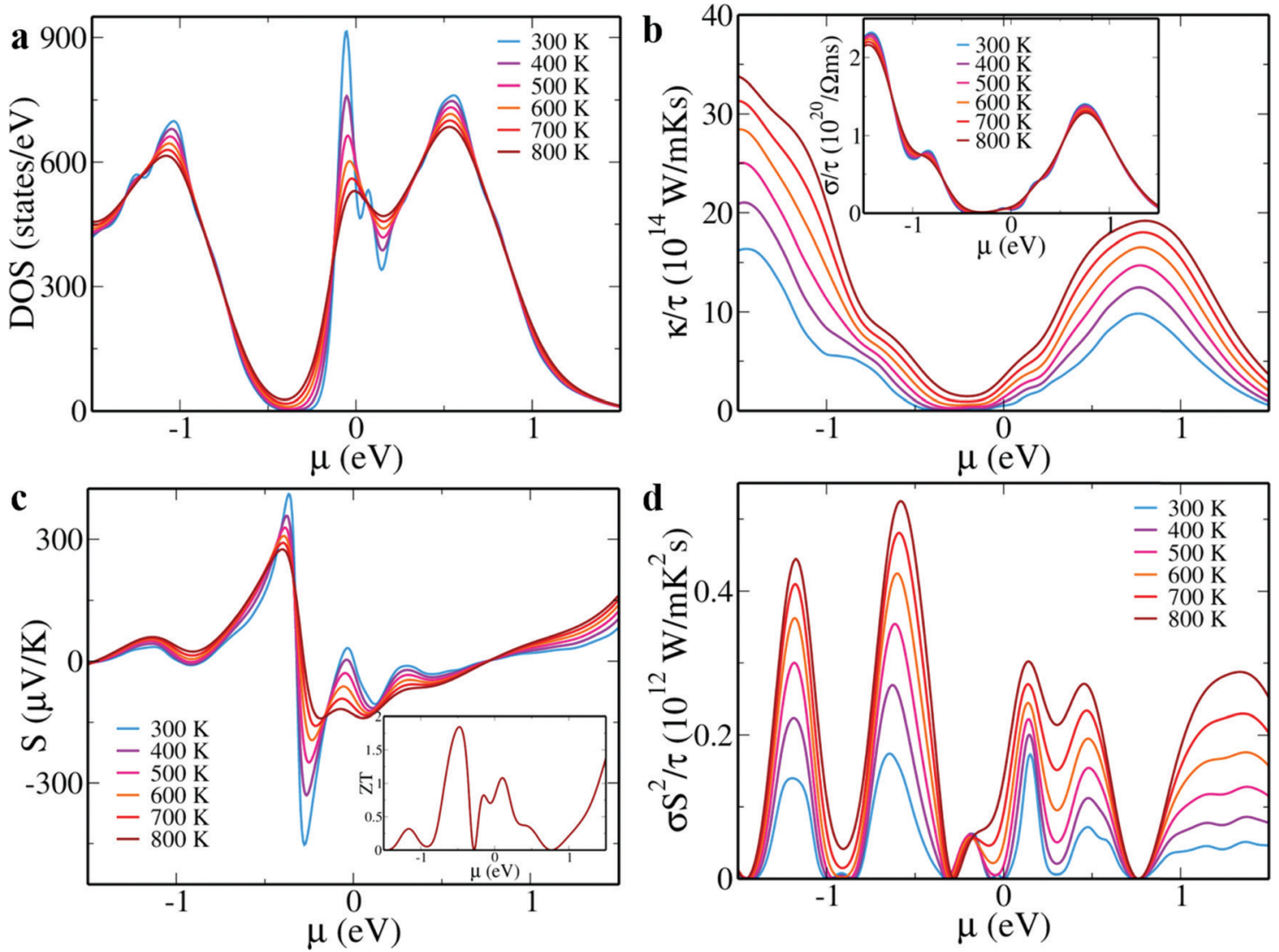

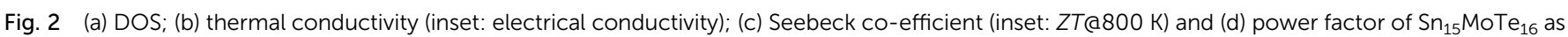
functions of chemical potential $(\mu)$ at various temperatures. Conductivity and power factor values are reported by scaling them with $\tau$. 
resonance levels, increasing the band effective mass and in turn the ' $S$ ' value. ${ }^{18}$

In undoped SnTe, the second set of eight-fold degenerate conduction bands $\left(L_{\mathrm{CB} 2}\right)$ lies $0.37 \mathrm{eV}$ above the first set of eightfold degenerate bands $\left(L_{\mathrm{CB}}\right)$. The interesting feature in Mo doped SnTe is the Rashba splitting of $L_{\mathrm{CB} 2}$ induced by strong spin-orbit coupling. ${ }^{18,38}$ The original single band edge splits into two band extrema $\left(\mathrm{CV}_{5}\right.$ and $\left.\mathrm{CV}_{6}\right)$, leading to an offset in the momentum $\left(k_{0}\right)$ by 0.09 and a Rashba energy $\left(E_{\mathrm{R}}\right)$ of $0.12 \mathrm{eV}$. The two parabolic branches with opposite spin aligned states occur as a consequence of specific atomic site asymmetries on either side of the $\Gamma$ point just $0.09 \mathrm{eV}$ above the Fermi level. ${ }^{18,38-40}$ We observe the effect of this splitting on the fourth set of doubly degenerate bands (of $L_{\mathrm{CB}}$ ) as well, which shows three valleys: $\mathrm{CV}_{2}$ at the $\Gamma$ point $0.02 \mathrm{eV}$ above the Fermi level, $\mathrm{CV}_{3}$ at $\Gamma+\delta^{\prime}$ in the $\Gamma \rightarrow Z$ direction $0.04 \mathrm{eV}$ above the Fermi level and $\mathrm{CV}_{4}$ at $\Gamma-\delta^{\prime \prime}$ in the $\Gamma \rightarrow \mathrm{M}$ direction $0.06 \mathrm{eV}$ above the Fermi level. Thus, Mo doping in SnTe provides a new approach for the generation of multiple bands for transport which lie in the close vicinity of the Fermi level, resulting in increased ' $S$ ' values. This also compensates for the decrease in carrier mobility caused by the resonance states. ${ }^{41}$ n-type dopants like Bi or p-type dopants like In and $\mathrm{Zn}$ in SnTe exhibit spin degenerate parabolic bands with single maxima. ${ }^{20,22,24}$ Hence, the defect mediated Rashba engineering in the conduction band is unique to the Mo resonant dopant as a consequence of its low atomic mass which induces local strain. ${ }^{40}$

We determined the DOS, ' $\sigma$ ', ' $S$ ', ' $\sigma S^{2}$ ' and ' $\kappa$ ' as functions of temperature and chemical potential ' $\mu$ ' using a rigid band approximation (Fig. 2). BoltzTraP code was used to implement the semiclassical theory under a constant scattering time approximation. ${ }^{42}$ At ' $\mu$ ' $=0$, the DOS revealed a peak due to the resonance level, the intensity of which decreased with the increase in temperature. ${ }^{43}$ While ' $\sigma$ ' and ' $\kappa$ ' showed similar variations with change in ' $\mu$ ' from the $\mathrm{p}$ - to n-type, the ' $\kappa$ ' values showed prominent increases in the values with increase in temperature. ${ }^{44}$ The sign of ' $S$ ' changed from positive to negative as we moved from the negative potential region corresponding to hole doping to the positive potential region corresponding to electron doping. ${ }^{43}$ Large positive and negative ' $S$ ' values indicate the success of tuning the electronic structure by doping Mo. The power factor shows 5 prominent peaks, with higher values in the p-type region. Assuming a lattice thermal conductivity of $0.4 \mathrm{~W} \mathrm{~m}^{-1} \mathrm{~K}^{-1}$ we estimated $Z T$ values of $\sim 1.84$ and $\sim 1.1$ at $800 \mathrm{~K}$ in the negative and positive potential regions, respectively. This indicates that the material can act as both $\mathrm{p}$ and n-type by tuning its chemical potential. ${ }^{18}$ The predicted p-type $Z T$ is much higher than the recently predicted $Z T$ of 1.66 for V doped SnTe exhibiting similar features. ${ }^{18}$ Implementing various strategies to reduce the lattice thermal conductivity further may lead to increases in the $Z T$ values, resulting in a promising thermoelectric material. ${ }^{19,45,46}$

\section{Conclusions}

In this work we have reported the versatile nature of molybdenum in improving the thermoelectric properties of SnTe.
A promising $Z T$ of $\sim 1.84$ and $\sim 1.1$ at $800 \mathrm{~K}$, when Mo acts as a p- and an n-type dopant, respectively, is predicted due to the tuning of the electronic structure. The increase in the power factor at low temperature is attributed to the formation of resonance levels. The bipolar transport is eliminated by the increase in the band gap. The convergence of light and heavy carrier valence and conduction bands increases the Seebeck coefficient for the $\mathrm{p}$ - and n-type materials respectively. The decrease in the electrical conductivity due to the low mobility of the carriers due to resonance scattering is more than compensated for by an increase in the number of contributing valleys due to multiband transport. The Rashba splitting of conduction bands causes several valleys to contribute to transport properties close to the Fermi level. This work unlocks the door for experimentalists to develop new thermoelectric materials with high performance.

\section{Conflicts of interest}

There are no conflicts of interest to declare.

\section{Acknowledgements}

The authors gratefully acknowledge the financial support received from CSIR, Govt. of India, in the form of R\&D project grants and DST, Govt. of India, for an INSPIRE Faculty Award.

\section{References}

1 X. L. Shi, J. Zou and Z. G. Chen, Advanced Thermoelectric Design: From Materials and Structures to Devices, Chem. Rev., 2020, 120, 7399-7515.

2 U. S. Shenoy and D. K. Bhat, Improving ZT of SnTe by Electronic Structure Engineering: Unusual Behavior of $\mathrm{Bi}$ dopant in the Presence of $\mathrm{Pb}$ as a Co-dopant, Mater. Adv., 2021, 2, 6267-6271.

3 Z. Chen, X. Guo, F. Zhang, Q. Shi, M. Tang and R. Ang, Routes for Advancing SnTe Thermoelectrics, J. Mater. Chem. A, 2020, 8, 16790-16813.

4 Y. Zhang, J. Sun, J. Shuai, X. Tang and G. Tan, Lead-free SnTe-based Compounds as Advanced Thermoelectrics, Mater. Today Phys., 2021, 19, 100405.

5 S. U. Shenoy and D. K. Bhat, Electronic Structure Engineering of $\mathrm{SrTiO}_{3}$ via Rhodium doping: A DFT Study, J. Phys. Chem. Solids, 2021, 148, 109708.

6 M. Li, Q. Sun, S.-D. Xu, M. Hong, W.-Y. Lyu, J.-X. Liu, Y. Wang, M. Dargusch, J. Zou and Z. G. Chen, Optimizing Electronic Quality Factor toward High-Performance $\mathrm{Ge}_{1-x-y} \mathrm{Ta}_{x} \mathrm{Sb}_{y} \mathrm{Te}$ Thermoelectrics: The Role of Transition Metal Doping, Adv. Mater., 2021, 33, 2102575.

7 S. Shenoy and D. K. Bhat, Enhanced Bulk Thermoelectric Performance of $\mathrm{Pb}_{0.6} \mathrm{Sn}_{0.4}$ Te: Effect of Magnesium Doping, J. Phys. Chem. C, 2017, 121, 20696-20703.

8 J. O. Sofo and G. D. Mahan, Optimum Band Gap of a Thermoelectric Material, Phys. Rev. B: Condens. Matter Mater. Phys., 1994, 49, 4565-4570. 
9 W. G. Zeier, A. Zevalkink, Z. M. Gibbs, G. Hautier, M. G. Kanatzidis and G. J. Snyder, Angew. Chem., Int. Ed., 2016, 55, 6826-6841.

10 U. S. Shenoy and D. K. Bhat, Electronic Structure Modulation of $\mathrm{Pb}_{0.6} \mathrm{Sn}_{0.4}$ Te via Zinc Doping and Its Effect on the Thermoelectric Properties, J. Alloys Compd., 2021, 872, 159681.

11 S. X. Lin, X. Tan, H. Shao, J. Xu, Q. Wu, G. Q. Liu, W. H. Zhang and J. Jiang, Ultralow Lattice Thermal Conductivity in SnTe by Manipulating the Electron-phonon Coupling, J. Phys. Chem. C, 2019, 123, 15996.

12 A. Banik, U. S. Shenoy, S. Saha, U. V. Waghmare and K. Biswas, High Power Factor and Enhanced Thermoelectric Performance of SnTe-AgInTe ${ }_{2}$ : Synergistic Effect of Resonance Level and Valence Band Convergence, J. Am. Chem. Soc., 2016, 138, 13068-13075.

13 U. S. Shenoy and D. K. Bhat, Selective Co-doping Improves the Thermoelectric Performance of SnTe: An Outcome of Electronic Structure Engineering, J. Alloys Compd., 2022, 892, 162221.

14 R. A. R. A. Orabi, N. A. Mecholsky, J. Hwang, W. Kim, J. S. Rhyee, D. Wee and M. Fornari, Band Degeneracy, Low Thermal Conductivity, and High Thermoelectric Figure of Merit in SnTe-CaTe Alloys, Chem. Mater., 2016, 28, 376-384.

15 G. Tan, L. D. Zhao, F. Shi, J. W. Doak, S. H. Lo, H. Sun, C. Wolverton, V. P. Dravid, C. Uher and M. G. Kanatzidis, High Thermoelectric Performance of p-type SnTe via a Synergistic Band Engineering and Nanostructuring Approach, J. Am. Chem. Soc., 2014, 136, 7006-7017.

16 D. K. Bhat and U. S. Shenoy, High Thermoelectric Performance of Co-Doped Tin Telluride Due to Synergistic Effect of Magnesium and Indium, J. Phys. Chem. C, 2017, 121, 7123-7130.

17 A. Banik, U. S. Shenoy, S. Anand, U. V. Waghmare and K. Biswas, Mg Alloying in SnTe Facilitates Valence Band Convergence and Optimizes Thermoelectric Properties, Chem. Mater., 2015, 27, 581-587.

18 U. S. Shenoy and D. K. Bhat, Vanadium: A Protean Dopant in SnTe for Augmenting its Thermoelectric Performance, ACS Sustain, Chem. Eng., 2021, 9, 13033-13038.

19 Z. Chen, Q. Sun, F. Zhang, J. Mao, Y. Chen, M. Li, Z. G. Chen and R. Ang, Mechanical Alloying Boosted SnTe Thermoelectrics, Mater. Today Phys., 2021, 17, 100340.

20 U. S. Shenoy and D. K. Bhat, Electronic Structure Engineering of Tin Telluride Through Co-Doping of Bismuth and Indium for High Performance Thermoelectrics: A Synergistic Effect Leading to Record High Room Temperature ZT in Tin Telluride, J. Mater. Chem. C, 2019, 7, 4817-4821.

21 Q. Zhang, B. Liao, Y. Lan, K. Lucas, W. Liu, K. Esfarjani, C. Opeil, D. Broido, G. Chen and Z. Ren, High Thermoelectric Performance by Resonant Dopant Indium in Nanostructured SnTe, Proc. Natl. Acad. Sci. U. S. A., 2013, 110, 13261-13266.

22 D. K. Bhat and U. S. Shenoy, Zn: A Versatile Resonant Dopant for SnTe Thermoelectrics, Mater. Today Phys., 2019, 11, 100158.

23 S. K. Kihoi, J. N. Kahiu, H. Kim, U. S. Shenoy, D. K. Bhat, S. Yi and H. S. Lee, Optimized Mn and Bi Co-Doping in SnTe
Based Thermoelectric Material: A Case of Band Engineering and Density of States Tuning, J. Mater. Sci. Technol., 2021, 85, 76-86.

24 U. S. Shenoy and D. K. Bhat, Bi and Zn Co-doped SnTe Thermoelectrics: Interplay of Resonance Levels and Heavy Hole Band Dominance Leading to Enhanced Performance and A Record High Room Temperature ZT, J. Mater. Chem. C, 2020, 8, 2036-2042.

25 G. Wu, Z. Guo, Q. Zhang, X. Wang, L. Chen, X. Tan, P. Sun, G. Q. Liu, B. Yu and J. Jiang, Refined Band Structure Plus Enhanced Phonon Scattering Realizes Thermoelectric Performance Optimization in CuI-Mn Co-doped SnTe, J. Mater. Chem. A, 2021, 9, 13065-13070.

26 S. K. Kihoi, U. S. Shenoy, D. K. Bhat and H. S. Lee, Complimentary Effect of Co-doping Aliovalent Elements $\mathrm{Bi}$ and $\mathrm{Sb}$ in Self-compensated SnTe-based Thermoelectric Materials, J. Mater. Chem. C, 2021, 9, 9922-9931.

27 S. K. Kihoi and H. S. Lee, Nanostructuring SnTe to Improve Thermoelectric Properties through $\mathrm{Zn}$ and Sb Co-doping, Sus, Energy Fuels, 2020, 4, 5645-5653.

28 D. K. Bhat and U. S. Shenoy, SnTe Thermoelectrics: Dual Step Approach for Enhanced Performance, J. Alloys Compd., 2020, 834, 155181.

29 S. Roychowdhury, S. U. Shenoy, U. V. Waghmare and K. Biswas, An enhanced seebeck coefficient and high thermoelectric performance in p-type In and $\mathrm{Mg}$ co-doped $\mathrm{Sn}_{1-\mathrm{x}} \mathrm{Pb}_{\mathrm{x}} \mathrm{Te}$ via the co-adjuvant effect of the resonance level and heavy hole valence band, J. Mater. Chem. C, 2017, 5, 5737-5748.

30 D. K. Bhat and U. S. Shenoy, Enhanced Thermoelectric Performance of Bulk Tin Telluride: Synergistic Effect of Calcium and Indium Co-Doping, Mater. Today Phys., 2018, 4, 12-18.

31 T. Hussain, X. Li, M. H. Danish, M. U. Rehman, J. Zhang, D. Li, G. Chen and G. Tang, Realizing High Thermoelectric Performance in Eco-friendly SnTe via Synergistic Resonance Levels, Band Convergence and Endotaxial Nanostructuring with $\mathrm{Cu}_{2}$ Te, Nano Energy, 2020, 73, 104832.

32 J. N. Kahiu, U. S. Shenoy, S. K. Kihoi, H. Kim, S. Yi, D. K. Bhat and H. S. Lee, Optimized Electronic Performance in Half-Heusler Ti-doped NbFeSb Materials by Stoichiometric Tuning at the Fe and Sb Sites, J. Alloys Compd., 2022, 891, 162033.

33 P. Giannozzi, S. Baroni, N. Bonini, M. Calandra, R. Car, C. Cavazzoni, D. Ceresoli, G. L. Chiarotti, M. Cococcioni and I. Dabo, et al., Quantum ESPRESSO: A Modular and OpenSource Software Project for Quantum Simulations of Materials, J. Phys.: Condens. Matter, 2009, 21, 395502.

34 J. P. Perdew, K. Burke and M. Ernzerhof, Generalized Gradient Approximation Made Simple, Phys. Rev. Lett., 1996, 77, 3865.

35 X. Wei, B. Zhao, J. Zhang, Y. Xue, Y. Li and Z. Yang, Chern Insulators without Band Inversion in $\mathrm{MoS}_{2}$ Monolayers with 3d adatoms, Phys. Rev. B, 2017, 95, 075419.

36 P. Guss, M. E. Foster, B. M. Wong, F. P. Doty, K. Shah, M. R. Squillante, U. Shirwadkar, R. Hawrami, J. Tower and D. Yuan, Results for Aliovalent Doping of $\mathrm{CeBr}_{3}$ with $\mathrm{Ca}^{2+}$, J. Appl. Phys., 2014, 115, 034908. 
37 D. K. Bhat and U. S. Shenoy, Mg/Ca Doping Ameliorates the Thermoelectrics Properties of GeTe: Influence of Electronic Structure Engineering, J. Alloys Compd., 2020, 834, 155989.

38 M. Hong, W. Lyv, M. Li, S. Xu, Q. Sun, J. Zou and Z. G. Chen, Rashba Effect Maximizes Thermoelectric Performance of GeTe Derivatives, Joule, 2020, 4, 2030-2043.

39 X. Zhang, Q. Liu, J. W. Luo, A. J. Freeman and A. Zunger, Hidden Spin Polarization in Inversion-symmetric Bulk Crystals, Nat. Phys., 2014, 10, 387-393.

40 X. Li, Y. Sheng, L. Wu, S. Hu, J. Yang, D. J. Singh, J. Yang and W. Zhang, Defect-mediated Rashba Engineering for Optimizing Electrical Transport in Thermoelectric BiTeI, npj Comput. Mater., 2020, 6, 107.

41 D. K. Bhat and U. S. Shenoy, Resonance Levels in GeTe Thermoelectrics: Zinc as a New Multifaceted Dopant, New J. Chem., 2020, 44, 17664-17670.

42 G. K. H. Madsen and D. J. Singh, BoltzTrap. A code for Calculating Band Structure Dependent Quantities, Comput. Phys. Commun., 2006, 175, 67-71.
43 U. S. Shenoy and D. K. Bhat, Vanadium Doped $\mathrm{BaTiO}_{3}$ as High Performance Thermoelectric Material: Role of Electronic Structure Engineering, Mater. Today Chem., 2020, 18, 100384.

44 U. S. Shenoy and D. K. Bhat, Enhanced Thermoelectric Properties of Vanadium Doped $\mathrm{SrTiO}_{3}$ : A Resonant Dopant Approach, J. Alloys Compd., 2020, 832, 154958.

45 F. Guo, B. Cui, C. Li, Y. Wang, J. Cao, X. Zhang, Z. Ren, W. Cai and J. Sui, Ultrahigh Thermoelectric Performance in Environmentally Friendly SnTe Achieved through StressInduced Lotus-Seedpod-Like Grain Boundaries, Adv. Funct. Mater., 2021, 31, 2101554.

46 D. An, J. Wang, J. Zhang, X. Zhai, Z. Kang, W. Fan, J. Yan, Y. Liu, L. Lu, C. Jia, M. Wuttig, O. CojocaruMiredin, S. Chen, W. Wang, G. J. Snyder and Y. Yu, Retarding Ostwald ripening through Gibbs adsorption and interfacial complexions leads to high-performance SnTe thermoelectrics, Energy Environ. Sci., 2021, 14, 5469-5479. 